\title{
Sur une opération pilote de prophylaxie antibilharzienne réalisée avec le dimethyldithiocarbamate de zinc (Zirame)
}

\author{
por S. GRÉTILLAT ef A. LACAN
}

La bilharziose génito-urinaire humaine, est une helmınthiase très répandue au Sénégal, où dans certaines régions (Haute et Basse Casamance, Sénégal oriental, Saloum), elle peut être considérée comme une des principales endémies.

Comme viennent de le démontrer les recherches poursuivies au Laboratoire national de recherches vétérinaires de Dakar-Hann sur le cycle évolutif des schistosomes, de l'Ouest-africain, l'agent causal de cette helminthıse est Schistosoma curassoni Brumpt, 1931, parasite commun à l'homme ef aux ruminants domestiques. Cette affection devant être considérée comme une amphixénose, le traitement des malades ne peut avoir qu'une très faible incidence sur l'abaissement du taux d'endémicité puisque le rumınant domestique représente un énorme réservoir de parasites et un disséminateur d'œufs du trématode ( 7,8 et 9 ).

Les mollusques hôtes intermédicires de $5 \mathrm{ch}$. curossoni, sont Bulinus guernei Dautzemberg au Sénégal oriental, dans le Saloum et la région du bas-fleuve Sénégal, B. lousseaumei Dautzemberg. en Casamance et B. truncatus rohifsi (Clessin) sur le plateau du Tagant en Mauritanie (2).

L'épidémiologie de la maladie étant en partie connue, ainsi que les conditions et lieux d'infestation des malades, il est normal que l'on ait songé à détruire l'hôte intermédiaire vecteur, chaînon indispensable au développement, à la

Rev. Elev. Méd. vét. Pays trop., 1963, no 2.

Reçu pour publication : févrıer 1963. multıplication du parasite et à 'sa dispersion.

(Utilisation de produits molluscicides détruisant la faune malacologique de certains marigots ou mares reconnus comme étant des foyers d'infestation.)

C'est ainsi que dans le cadre des recherches entreprises par le service d'Helminthologie du laboratoire national de l'Elevage et de recherches vétérinaires de Dakor au sujet de la prophylaxie des maladies parasitaires à trématodes, s'inscrivent les essais à effectuer sur le terrain avec un nouveau produit molluscicide testé et mis au point ou cours des années 1960 et 1962, par le même établissement de recherches, en l'occurence de diméthyldithiocarbamate de zinc ou Zirame (3-4-5-6, 10 et 11).

D'autre part, le service des grandes endémies du Ministère de la Santé et des affaires sociales de la République du Sénégal désırant entreprendre au cours de l'année 1963 plusieurs compagnes de prophylaxie antibilharzienne par destruction des mollusques hôtes intermédiaires, une opération pilote à l'aide du diméthyldithiocarbamate de zinc fut réalisée dans les gîtes à mollusques de la région de Tambacounda (Sénégal oriental).

Cette région fut chossie pour les raisons suivantes :

a) Dans la partie du Sénégal située au nord-est, au nord, au nord-ouest, et au sud-ovest de Tambacounda, la nature des gîtes à mollusques vecteurs est connue depuis les prospections malacologiques effectuées en 1961, sur la de- 
mande du service des grandes endémies du Ministère de la Santé et des affaires sociales de la République du Sénégal, par le laboratore national de recherches vétérinaires de Dakar (2).

b) L'importance moyenne des points d'eau à traiter et leur diversité ont permis' d'opérer dans des conditions très intéressantes au point de vue de la mise au point des techniques d'épandage du produit anti-mollusques, ainsi que des solutions à trouver aux problèmes posés par son transport sur les lieux d'utilisation qui sont. parfois très difficiles d'accès.

c) Les foyers d'infestation des habitants et des animaux étant très nettement circonscrits dans cette région (mares et marigots permanents ou semi permanents), les contrôles d'efficacité se sont avérés beaucoup plus faciles à réaliser que dans dès points d'eau de très grandes dimensions ef moins bien délimités.

d) La diversité des gîtes à traiter a permis de mettre au point et de comparer les diverses méthodes à utiliser pour l'évaluation du volume de l'eau à assainir. e) Travaillant sur des mares ou des biefs de marigot isolés les uns des autres, il a été possible de déterminer les doses minima mortelles pour les gastéropodes dans les conditions de la pratique courante, tout en évaluant l'incidence du traitement sur, le devenir de la faune et de la flore du milieu.

f) Cette opération de moyenne envergure a permis de chiffrer le coût approximatif d'une campagne de'plus grande importance sans entraîner de gros frais dus aux erreurs toujours possibles au cours d'une expérimentation à grande échelle.

En résumé, cette campagne pilote de lutte antimollusques s'est déroulée sous forme d'essais présentant d'une part, un intérêt au point de vue recherche, et d'autre part, marquant un pas vers le stade de prévulgarisation des techniques ef procédés à employer dans l'utilisation du produit molluscicide sur une grande échelle, avec des moyens beaucoup plus importants.

Enfin, Il a semblé nécessaire de connaître (et ce point est loın d'être négligeable) les réac-

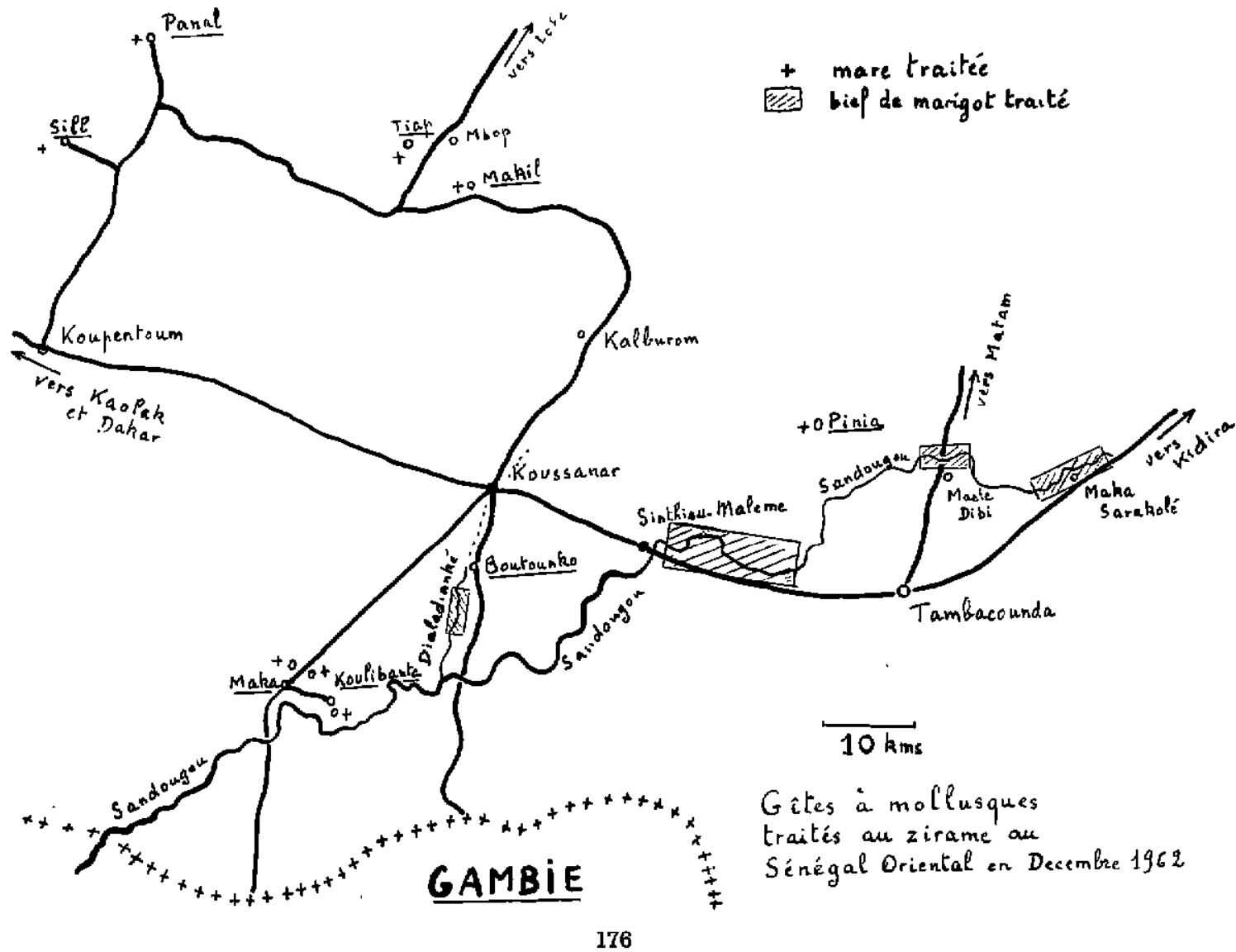


trons des habitants utilisant les points d'eau traités pour leur usage personnel (boisson, baignade, lessive) et l'abreuvement de leurs animaux domestıques, avant de se lancer dans l'assainissement de toute une région.

\section{PLAN DE TRAVAIL}

\section{A. - Enquête préliminaire}

a) Repérage des points d'eau avec leur importance et leur niveau permettant de fixer la date d'intervention la plus adéquate.

En effet, compte tenu de l'écologie du bulin vecteur rencontré dans cette région, qui s'enfonce dans le fond vaseux des mares et marigots quelques semaines avant leur déssèchement complet, il y a lieu d'appliquer le produit molluscicide quelques semaines avant cette période de migration, les mollusques enfoncés dans la vase ne pouvant être atteints par le produit. D'autre part, pour des raisons de prix de revient, if est recommandé de ne traiter qu'un volume d'eau réduit. Le choix judicieux de la date d'épandage du molluscicide présente donc un grand intérêt pratique et économique.

b) Etablissement du plan de campagne sur le terrain et sur carte, pour chiffrer et évaluer :

10 les distances et les moyens d'accéder aux gîtes à traiter, depuis un centre de rayonnement où sont entreposés matériels et produit antimollusques.

$2^{\circ}$ en tenant compte de la nature, de l'importance et des conditions d'environnement des différents points d'eau, prévoir pour chacun d'eux, une technique de traitement approprié et déterminer leur volume approxımatif afin de chiffrer les quantités de molluscicide nécessaires à l'opération.

\section{B. - Opération pilote proprement dite}

a) Calcul du volume d'eau d̀ traiter et de la quantité de molluscicide à répandre, soit par unité de surface (mare) soit par unité de longueur de gîte (marigot), compte tenu naturellement de la profondeur et de la largeur du cours d'eau à traiter. b) Mesure du pH de l'eau, nature du fond, de la flore et de la faune aquatique.

c) Epandage du produit molluscicide.

d) Mise en place de «bâtons-pièges» ou de «cages de contrôle» pour évaluer l'efficacité et la diffusion du produit.

e) Contrôle des résultats obtenus au point de vue efficacité, diffusion, et toxicité du produit.

\section{C. - Contrôles de fin d'expérimentation}

Faits 10 d̀ 15 jours après l'opération pilote proprement dite.

Evaluation du taux de mortalité parmı la faune malacologique.

Modifications éventuelles de la faune et de la flore aquatiques par observations et contrôles directs (informations recueilites auprès des habitants utilisant les points d'eau traités).

Réactions et attitude des villageois ef des pasteurs transhumants.

\section{MATÉRIEL ET MOYENS DE TRANSPORT UTILISÉS}

Le produit molluscicide utilisé pour cette opération pilote fut à une exception près (mare de Sili, traitée au Bayer 73), du diméthyldithiocarbamate de zınc ou Zirame, présenté sous forme de poudre micronisée titrant $90 \%$ de produit pur, dont $90 \%$ au mains de partıcules ont un diamètre inférieur à $10 \mu$.

Cettle poudre très légère est emballée en sacs de $20 \mathrm{~kg}$ d'un volume approximatif de $80 \mathrm{dm}^{3}$, en jute, doublé d'un emballage en polyéthylène.

Le Zirame, qui a une solubilité maximum de $65 \mathrm{mg} / \mathrm{l}$ est un produit de synthèse ne présentant aucun danger pour les utilisateurs. Les seules précautions à prendre consistent à éviter son contact avec les muqueuses qu'il irrite (éternuements et picotements) et avec les yeux (larmoiement).

Les expériences faites en 1960-1961 au laborataire national de l'Elevage et de recherches vétérinaires de Dakar et dans quelques points d'eau, ont montré qu'il avait une activité molluscicide très marquée sur Bulinus guernei Dautzemberg, Bulinus senegolensis (Müller) Bromphaiaria 
pfeiffer gaudi Ranson et Lymnaea natalensis coilloudı Bourguignat, à des doses variant entre 1 à 2 parties par million. II possède, d'autre part, un pouvoir rémanent de trois semaines à un mois dans des milieux très vaseux quand on l'utilise à des doses de 5 à 10 p. p. m. (5 à $10 \mathrm{~g} / \mathrm{m}^{3}$ ).

Sous forme de poudre micronisée, il diffuse très bien même dans des milieux encombrés de plantes aquatiques et il n'esł que lentement absorbé par les matières organiques.

Autre propriété intéressante, il tue les larves de Culicidae à des doses de 1 à 2 p.p. m., ce qui permet de grouper en une seule intervention les prophylaxies antibilharzienne et antipalustre (6).

\section{Main-d'œuvre.}

Pour l'assainissement des mares et des marigots de la région proche de Tambacounda, quatre mancuvres ont été employés pendant trols jours et demi. Pour l'opération accomplie en région de Koussanar, deux mancuvres engagés sur place pour une durée de trois jours permirent d'effectuer tout le travail courant (transport des sacs, arpentage des contours des mares, gonflage du canat pneumatique, etc...).

Quant à l'épandage du molluscicide, il a été confié, pour les marigots, à un aide de laboratoire, qui s'acquitta très bien de cette tâche, délicate si l'on considère que la quantité du produit à répandre varie suivant la largeur ef la profondeur du cours d'eau.

\section{Epandage du produit anti-mollusque.}

La phase d'épandage du molluscicide doit être précédée par le calcul du volume des eaux à traiter.

A ce sujet, dans la région où eut lieu cette opération pilote, deux catégories de points d'eau, les mares et les biefs de marigots posèrent des problèmes différents au point de vue de l'évaludtion de leur volume, le facteur débit n'intervenant pas étant donné qu'il s'agissait d'une opération en eau dormante.

a) Mares.

Ce sont des collections d'eau à bords plus ou moins irréguliers, à fond latéritique en général, régulièrement incliné vers la partie la plus profonde de la mare là où se trouvent la où les résurgences, dont le sol est la plupart du temps très vaseux, reconnaissables en surface grâce à une flore beaucoup plus dense de Nymphoea ef parfois de lotus.

Ces mares étant à cette époque de l'année de petite superficie $\left(2.000\right.$ à $\left.5.000 \mathrm{~m}^{2}\right)$, il a été possible d'établir leur profondeur moyenne en envoyant dans leurs différents points, un sondeur équipé de grandes bottes de chasse. La profondeur maximum de certaines mares peut atteindre plus d'1,50 m, alors que la profondeur moyenne reconnue à l'entour varie généralement entre $0,30 \mathrm{~m}$ et $0,50 \mathrm{~m}$.

Le calcul de la superficie de ces points d'eau fut beaucoup plus malasé en raison des contours irréguliers des bords, présentant parfois des diverticules latéraux de plus ou monns grande importance. Si ces derniers étaient étendus, et nettement circonscrits, on procédait à l'évaluation de leur volume en les considérant comme des mares latérales. S'ils étaient de superficie réduite, le contour de la mare était jalonné de telle sorte que sa surface soit représentée par un polygone simple dont les côtés suivent ou coupent les bords irréguliers de la collection d'eau, en veillant à ce que dans la mesure du possible, la surface des digitations compense approximativement celle des enclaves.

Ce piquetage permit de rapporter sur papier, après arpentage et relevé des angles à l'alidade, une figure dont il était facile de calculer la surface. Le volume était alors facile à obtenir.

Les chiffres trouvés étaient certes entachés d'erreurs, mais avaient cependant assez de valeur pour fixer la quantité de malluscicide à répandre.

Pour les mares, nous nous sommes contentés de répandre le Zirame à la main sur leur périphérie, le pouvoir de diffusion du produit étant largement suffisant pour qu'll se répartisse en quelques heures dans toute la masse aquatique.

b) Biefs de marigots.

Le principal marigot existant dans les régions Nord-ouest et sud-ovest de Tambacounda est un affluent du fleuve Gambie, le Sandougou. La longueur approximative de son cours est d'une centaine de km.

Les enquêtes malacologıques réalisées fin novembre 1962, ont montré que c'est le tiers supérieur de son cours qui récèle des gîtes per- 


\begin{tabular}{|c|c|c|c|c|c|c|c|c|c|}
\hline \multicolumn{10}{|c|}{ Tableau $n^{0} I$} \\
\hline \multicolumn{10}{|c|}{ Lutte antimollusques dans les mares } \\
\hline \multirow{2}{*}{$\begin{array}{l}\text { Nom der gites } \\
\text { traltés }\end{array}$} & \multirow{2}{*}{$\begin{array}{l}\text { Volume ou } \\
\text { importance } \\
\text { (en m3) }\end{array}$} & \multirow[t]{2}{*}{ Fiore } & \multirow{2}{*}{$\begin{array}{l}\text { Eau } \\
\text { pH }\end{array}$} & \multirow{2}{*}{ Fond } & \multicolumn{2}{|c|}{ MoIlusques (espècess et densité) } & \multirow{2}{*}{$\begin{array}{l}\text { Quant1té de } \\
\text { mo1luacicide }\end{array}$} & \multirow[t]{2}{*}{ Dose } & \multirow{2}{*}{$\begin{array}{c}\text { Résuitats } \\
\text { (mortalité des mollusques) }\end{array}$} \\
\hline & & & & & B.guerni & B.senegalensis & & & \\
\hline Panal & $60.000 \mathrm{~m} 3$ & $\begin{array}{c}\text { Nymphaea } \\
\text { rares }\end{array}$ & $\begin{array}{c}\text { trouble } \\
6,2\end{array}$ & arglleux & très rares * & & $\begin{array}{l}\text { Zijame } \\
60 \mathrm{~kg}\end{array}$ & 1 p.p.m. & 100 p.100 \\
\hline Maldi & $4.000 \mathrm{nz}$ & $\begin{array}{l}\text { Nymphees } \\
\text { et lotus } \\
\text { abondants }\end{array}$ & $\begin{array}{c}\text { claire } \\
6,0\end{array}$ & $\begin{array}{l}\text { latérit1- } \\
\text { que }\end{array}$ & 50 par m2 et plus & très rares & $\begin{array}{l}\text { Zirame } \\
30 \mathrm{~kg}\end{array}$ & 7 p.p.m. & $\begin{array}{l}\text { cadiavres observes au bord } \\
\text { it en surface }\end{array}$ \\
\hline THap & $3.600 \mathrm{m3}$ & $\begin{array}{l}\text { Mympheea } \\
\text { et lotus } \\
\text { Bbondants }\end{array}$ & $\frac{c l a t r e}{6,2}$ & $\begin{array}{l}\text { latériti- } \\
\text { que et } \\
\text { vaseux }\end{array}$ & 40 à $50 \mathrm{par} \mathrm{m} 2$ & 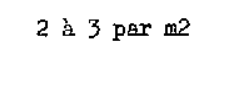 & $\begin{array}{l}\text { Zirame } \\
40 \mathrm{~kg}\end{array}$ & $12 \mathrm{p} \cdot p, \mathrm{~m}$. & $\begin{array}{l}\text { mare nan oontrôlée } \\
\text { (acod̀a très difflcilo) }\end{array}$ \\
\hline Pinia & $10 \underset{\mathrm{ta3}}{10} 11.000$ & $\begin{array}{l}\text { Nymphoes } \\
\text { lotus et } \\
\text { joncs ra- } \\
\text { res }\end{array}$ & $\begin{array}{c}\text { trouble } \\
6,2\end{array}$ & $\begin{array}{l}\text { latérit1- } \\
\text { que et } \\
\text { vaseux }\end{array}$ & 10 b 20 par m 2 & rares & $\begin{array}{l}\text { Z1rame } \\
25 \mathrm{~kg}\end{array}$ & 3 p.p.m. & $\begin{array}{l}\text { mare non controlée } \\
\text { (acces trob diffic1le) }\end{array}$ \\
\hline Malea (mare I) & $2.500 \mathrm{mz}$ & \begin{tabular}{|l} 
Nynphasea \\
abondents
\end{tabular} & $\begin{array}{c}\text { trouble } \\
6,2\end{array}$ & vaseux & 5 par m2 & - & $\begin{array}{l}21 \text { irame } \\
12,5 \mathrm{~kg}\end{array}$ & 5 p.p.m. & $100 \mathrm{p.100}$ \\
\hline Maka (mare II) & $1.800 \mathrm{~m} 3$ & \begin{tabular}{|l} 
Nymphaea \\
abondants
\end{tabular} & $\begin{array}{c}\text { vaseuse } \\
6,0\end{array}$ & $\begin{array}{l}\text { très } \\
\text { vaseux }\end{array}$ & 2 a $5 \mathrm{par}$ m 2 & - & $\begin{array}{l}21 \mathrm{ram} \theta \\
10 \mathrm{~kg}\end{array}$ & 5,5 p.p.m. & $100 \mathrm{p} .100$ \\
\hline Koulibanta & $2.400 \mathrm{~m} 3$ & $\begin{array}{l}\text { Nymphaes } \\
\text { assez ra- } \\
\text { reg }\end{array}$ & $\begin{array}{c}\text { vaseuse } \\
6,0\end{array}$ & $\begin{array}{l}\text { très } \\
\text { vaseux }\end{array}$ & moins de 5 par m2 & - & $\begin{array}{l}\text { Z1rame } \\
8,5 \mathrm{kgg}\end{array}$ & 3,5 p.p.m. & $100 \mathrm{p} \cdot 100$ \\
\hline Sil1 & $1.500 \mathrm{m3}$ & $\begin{array}{l}\text { Nymphaea } \\
\text { abondents }\end{array}$ & $\begin{array}{l}\text { trouble } \\
\text { et ve- } \\
\text { eeuse } \\
6.0\end{array}$ & $\begin{array}{l}\text { latériti- } \\
\text { que ot } \\
\text { vaseux }\end{array}$ & plus de 100 par m2 & rares & $\begin{array}{l}\text { Bayer } 73 \\
1.750 \mathrm{~kg}\end{array}$ & $\begin{array}{l}1 \stackrel{\text { a } 1,25}{\text { p.p.r. }} \\
\text { p.p. }\end{array}$ & $\begin{array}{l}\text { quelques mollusques en- } \\
\text { core vivants dems les } \\
\text { anfractuosités. }\end{array}$ \\
\hline
\end{tabular}

* Cette mare avast déjà été trajtáe on 1961. 


\begin{tabular}{|c|c|c|c|c|c|c|c|c|}
\hline & 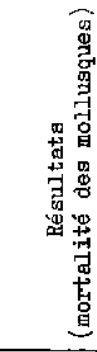 & 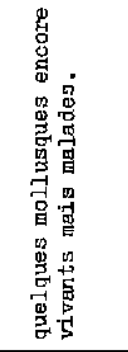 & $\begin{array}{l}\stackrel{8}{0} \\
\dot{a} \\
\underline{8}\end{array}$ & \begin{tabular}{l}
8 \\
\hdashline \\
$\dot{2}$ \\
8 \\
8
\end{tabular} & $\begin{array}{l}\stackrel{8}{0} \\
\stackrel{3}{0} \\
8 \\
8\end{array}$ & $\begin{array}{l}\stackrel{8}{0} \\
\dot{2} \\
\underline{8}\end{array}$ & $\begin{array}{l}\frac{8}{0} \\
\dot{2} \\
8 \\
\frac{8}{2}\end{array}$ & 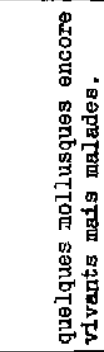 \\
\hline & 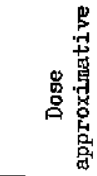 & $\begin{array}{l}\dot{\dot{a}_{0}} \\
\dot{\dot{\alpha}} \\
\dot{\dot{a}_{1}}\end{array}$ & $\begin{array}{l}\dot{a} \\
\dot{a} \\
\dot{a} \\
\sim\end{array}$ & $\begin{array}{l}\dot{\overline{\dot{a}}} \\
\dot{\dot{a}} \\
\dot{v} \\
\mathrm{v}\end{array}$ & $\begin{array}{l}\dot{9} \\
\stackrel{\dot{a}}{\dot{a}} \\
\sim \\
\sim\end{array}$ & $\begin{array}{l}\dot{j} \\
\stackrel{\dot{\alpha}}{\dot{\alpha}} \\
\sim \\
\sim\end{array}$ & $\begin{array}{l}\dot{a} \\
\dot{a} \\
\dot{a} \\
\dot{m} \\
m\end{array}$ & 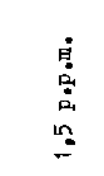 \\
\hline & 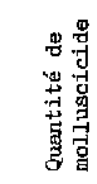 & 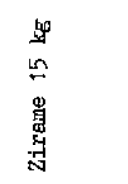 & 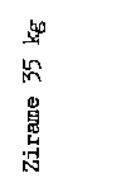 & 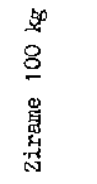 & 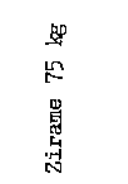 & 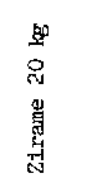 & 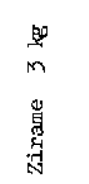 & 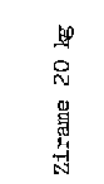 \\
\hline 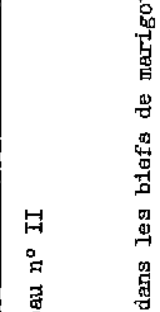 & 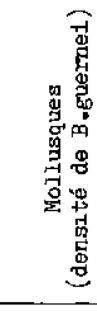 & 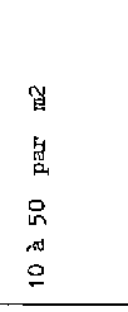 & 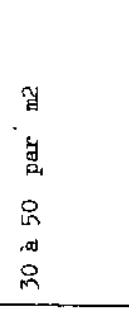 & 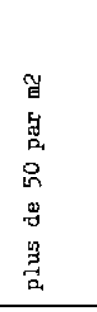 & 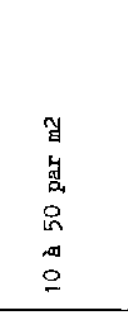 & 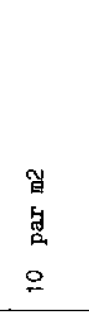 & 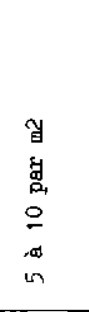 & $\begin{array}{l}\text { 염 } \\
\text { y } \\
\text { a } \\
\text { in } \\
\text { g } \\
3 \\
\text { g } \\
\text { ․ }\end{array}$ \\
\hline 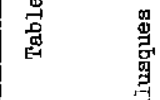 & 㟥 & 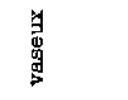 & 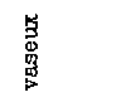 & 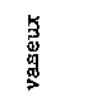 & 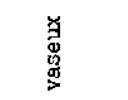 & 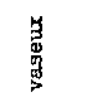 & 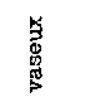 & 总害 \\
\hline 基 & 踾瞄 & $\tilde{b}^{n}$ & $\tilde{6}$ & $8^{\circ}$ & : & 气ू & مु & \\
\hline t & 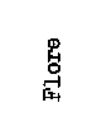 & 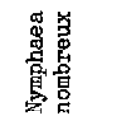 & 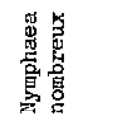 & 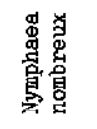 & 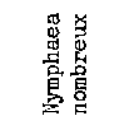 & 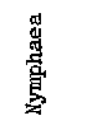 & 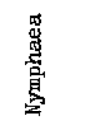 & 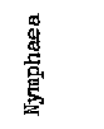 \\
\hline & 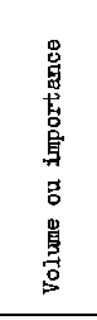 & 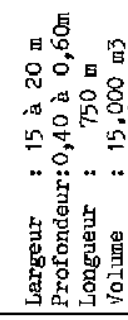 & 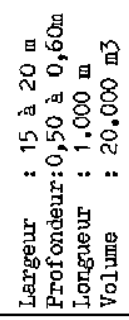 & 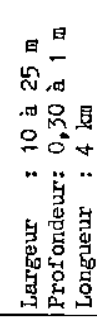 & 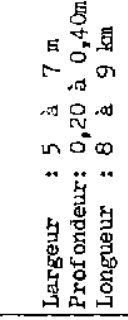 & $\begin{array}{l}\text { 简 } \\
\text { o } \\
\dot{0}\end{array}$ & 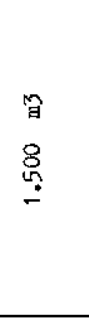 & 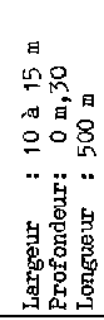 \\
\hline & 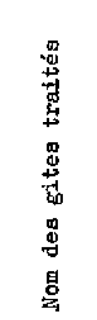 & 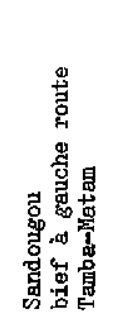 & 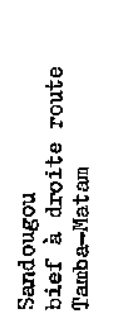 & 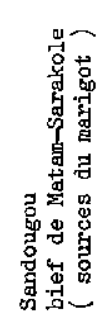 & 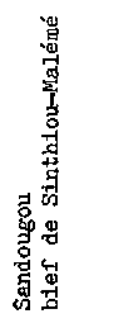 & 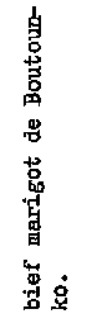 & 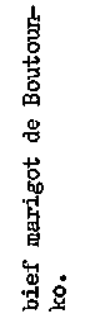 & 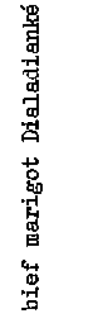 \\
\hline
\end{tabular}


manents à B. guernei : par suite de leur assèchement très précoce (octobre-début novembre) ses marigots affluents ne semblent pas permettre d̀ ce gastéropode d'eau douce «d'estiver» dans des conditions normales, dons des fonds sans doute trop éloignés de la nappe phréatique. Les très nombreuses coquilles vides de bulins trouvées sur le bord et sur le fond fissuré de ces petits cours d'eau, démontreraient aussi qu'un dessèchement précoce ef rapide tue les mollusques avant qu'ils n'aient eu le temps de s'enfouir dans la vase.

Le gîtes permanents à B. guernei sont donc en novembre/décembre, des biefs de marigots dont la longueur varie de quelques centaines de $\mathrm{m}$. à plusieurs $\mathrm{km}$ ayant de 10 à $20 \mathrm{~m}$ de large, sur 30 à $80 \mathrm{~cm}$ de profondeur, le plus souvent 40 à $50 \mathrm{~cm}$.

Ces grandes collections d'eau très vaseuse par endroits ( 20 à $30 \mathrm{~cm}$ de vase fluide), sont encombrées d'une épaisse végéfation aquatique comprenant principalement des nénuphars. Certaines, quoique de volume très réduit en fin de saison sèche, ne farissent que très exceptionnellementfin juin, début juillet. Ce sont des bas-fonds du lit du marigot maintenus en eau par les affleurements ou des résurgences, et séparés par des hautsfonds résultant de l'accumulation d'alluvions ef surtout de déblais résultant de l'érosion des flancs de la vallée.

Pour calculer le volume de l'eau à traiter, nous avons procédé de la manière suivante : mesure du profil moyen du marigot avec évaluation de la quantité d'eau par $m$ linéaire. A partir de ce volume initial, est établi le poids de Zirame à répandre par $m$ de cours d'eau. En cours d'opération des corrections en plus ou en moins sont apportées à ce chiffre selon que la largeur ef la profondeur du marigot augmentent ou diminuent.

Ces biefs de marigots n'étant en général abordables qu'en certains points (rives encombrées de broussailles et très boisées), il s'avéra peu pratique de tenter leur assainissement par épandage de Zirame à la main tout le long des rives. Un canot pneumatique fut donc utilisé : l'aide chargé de répandre et de doser le produit déversaif le Zirame dans l'eau au moyen d'une mesure préalablement jaugée.

Les tableaux I et Il résument les actions entreprises et les résultats obtenus.

\section{DISCUSSION ET INTERPRÉTATION DES RÉSULTATS OBTENUS} AU COURS DE CETTE OPÉRATION PILOTE

\section{Assainissement des mares.}

Les résultats obtenus dans les mares de PANAL, MAKIL, MAKA, MAKA II et KOULIBANTA, démontrent que, dans les conditions rencontrées sur le terrain, une dose de 2 à 3 p. p. m. de diméthyldithiocarbamate de zinc est suffisante pour tuer tous les bulins présents dans un gîte.

Le pouvoir de diffusion du produit permet de ne le répandre qu'à la périphérie du point d'eau, le Zirame atteignant la partie centrale, soit par flattation de la poudre à la surface de l'eau, soit par simple dilution.

Expérimentalement, des cages en bois de $30 \mathrm{~cm}$ d'arête, recouvertes de treillis de nylon, dans lesquelles nous avions placé une cinquantaine de mollusques fixés sur des feuilles et des tiges de nénuphars nous ont permis d'évaluer le pouvoir de diffusion du Zirame en eau calme.

Les cages étaient disposées dans un coin de la mare de manière que leur emplacement soit distant d'une cinquantaine de $m$ du dernier endroit où le Zirame avait été répandu.

Les contrôles de mortalité faits 72 heures après montrèrent que tous les bulins étaient morts depuis au moins 24 heures (coquilles blanc grisâtre vides de leur contenu).

Les mêmes résultats furent obtenus dans des mares où les Nymphoeo et les lotus étaient très abondants. L'épaisseur ef la densité de la flore aquatique ne semblent gêner en aucune manière, la diffusion du produit.

\section{$2^{\circ}$ Assainissement des biefs de marigots.}

En tenant compie des erreurs de dosage toujours possibles, dues surtout à l'évaluation du volume de l'eau à traiter, si l'on considère les résultats obtenus dans les différents biefs de marigots, des doses égales ou supérieures à 3 p. p. m. sont nécessaires. En effet, l'épandage du diméthyldithiocarbamate de zinc $y$ est plus régulier que dans les mares en raison de la faible largeur des biefs, mais il y a lieu de tenir compte. dans l'évalution du volume de l'eau à traiter, de la grande quantité de vase fluide qui repose sur le 
fond et dilue le molluscicide dans de notables proportions.

Les contrôles d'efficacité ont été réalisés sur bâtons-pièges qui sont en général des fragments de branches d'arbres plus ou moins pourris sur lesquels aiment à se fixer les moliusques.

Cette technique de contrôle est certes beaucoup plus grossière que celle des cages, mais c'est la seule rédisable dans les points d'eau éloignés où le matériel laissé sur place est considéré comme abandonné et disparaît dans les jours qui suivent sa mise en place.

Les échecs partiels constatés dans les biefs du Sandougou (Route de Tamba-Matam, bief gauche) et du Dialadanké seraient ainsi imputables à la nature très vaseuse de leurs fonds.

\section{TOXICITÉ DU ZIRAME}

\section{lo Sur la flore aquatique.}

Aux doses utilisées au cours de cette opération pilote le diméthyldithiocarbamate de zinc n'a présenté aucun effet toxique sur les Nymphoea, les lotus et les Cypéracées, représentant la flore aquatique de ces mares et marigots.

\section{$2^{\circ}$ Sur la faune aquatique.}

a) Poissons, Les questions posées aux riverains des points d'eau traités (villageois ou pasteurs transhumants) lors des contrôles d'efficacité faits 15 jours environ après l'épandage du molluscicide, permettent d'affirmer que le diméthyldithiocarbamate de zinc ne détruit qu'en partıe la faune ichtyologique.

Les silures (Clarias sp.) sont épargnés; par contre les jeunes Hemichromis fasciatus, $\mathrm{H}$. bimaculatus et les Tilapia melonopleura sont plus sensibles à l'action du Zirame, sans toutefois être détruits en totalité comme nous avons pu le constater dans deux biefs de marigots, celul de Bautounko I (contrôlé quatre jours après l'épandage) et celui de Maka-Sarakolé où de nombreux poissons vivaient encore 15 jours après son assainissement.

b) Batraciens. Si les larves de grenovilles (tétards) sont fuées par le Zirame, it ne nous a pas été possible de retrouver des cadavres de batraciens le long des bords des mares et des marigołs traités. c) Insectes aquotiques. Les larves de libellules mises à part, les coléoptères aquatiques adultes ne semblent pas être détruits par le diméthyldithiocarbamate de zinc.

d) Mollusques aquatiques autres que les Bulins. Les mares de cette région présentent pour la plupart d'innombrables spécimens de Caelaturo mesafricana Pilsby et Bequaert, dont la cavité paliéale est souvent occupée par une larve commensale d'Ephéméroptère. Ce mollusque Eulamellibranche, comme son commensal, sont tués par le Zirame aux doses toxiques pour les $B$. guerner et $B$. senegalensis.

\section{ACTION MOLLUSCICIDE DU BAYER 73 OU BAYLUCIDE}

A titre de comparaison, nous avons essayé ce molluscicide dans une mare (mare de Sili) de faibles dimensions, les quantités de BAYER 73 que nous avions à notre disposition étant très faibles.

Cette collection d'eau, à fond latéritique, fut traitée à raison de 1 à 1,25 p. p. m., dose double de celle préconisée par la Maison BAYER ef par les chercheurs ayant procédé à des essais sur le terrain $(1 ; 12$ et 13$)$.

Pour évaluer le pouvoir de diffusion du produit, nous l'avons déposé le long du tiers de la périphérie de la mare à environ 20 à $35 \mathrm{~m}$ des points les plus éloignés.

Les contrôles d'efficacité faits 15 jours après permirent de constater :

Une mortalité de 100 p. 100 chez les B. guernei, $B$. senegalensis et Caelatura mesafricana. Cependant, dans quelques anfractuosités des bords de cette mare (poches d'eau communiquant avec la masse d'eau centrale par de petits diverticules latéraux), nous avons pu recueillir quelques $B$. guernel encore vivants. Le BAYER 73 devrait peut-être être répandu becucoup plus uniformément que le Zirame et aurait vraisemblablement un pouvoir de diffusion plus faible.

Malgré cela, on peut considérer ce produit comme diffusant bien même en equ calme, la présence de nombreux pieds de nénuphars n'entravant pas son action molluscicide en des points situés à plus de $30 \mathrm{~m}$ de son lieu d'épandage.

Toxicité pour la flore aquatique : Le BAYER 73 ne s'est pas révélé toxique pour les Nymphoea. 
Toxicité pour la faune aquatique : L'absence de poissons et de batraciens dans cette mare n'a pas permis d'évaluer son degré de toxicité pour ces vertébrés. Par contre, nous avons trouvé de très nombreux cadavres de larves de libellules et de coléoptères aquatiques à la surface de cette collection d'eau 14 jours après son traitement par le BAYER 73.

\section{ACCUEIL ET ATTITUDE DES HABITANTS UTILISANT LES POINTS D'EAU ASSAINIS}

Ces mares et la plupart des biefs de ces marigots sont utilisés par de très nombreux habitants pour le ravitaillement en eau de boisson, les ablutions, le lavage du linge et l'abreuvement des anımaux. L'épandage de molluscicide dans ces collections d'eau dont l'importance est vitale pour eux, aurait pu les amener à considérer une telle intervention comme contraire à leurs intérêts, et à nous soupçonner de polluer leurs seules ressources en eau avec un produit chimique peut-être nocif pour eux et pour leurs animaux. II n'en a rien été, au contraire.

Il a suffi de leur expliquer en termes simples que l'hématurie dont ils souffrent a pour origine la présence des mollusques dans les mares et marigots, et que leur destruction au moyen du Zirame ne peut qu'apporter une amélioration de l'état sanitaire de la région.

Lors des contrôles d'efficacité faits deux semaines après le traitement, nombreux furent les éleveurs venant nous annoncer la destruction massive des mollusques en nous montrant les nombreuses coquilles vides de bulins jonchant les bords des mares ou des marigots, et nous donnant spontanément les observations qu'ils avaient pu faire au sujet de la toxicité du produit sur les poissons.

\section{PRIX DE REVIENT DE CETTE OPÉRATION PILOTE ANTIMOLLUSQUE}

II serait fort présomptueux de prétendre qu'une seule intervention antimollusques sur le terrain suffit à abaisser consıdérablement le taux d'endémicité bilharzienne dans la région considérée.
Il y a lieu de prévoir, en effet, d'autres opérations au cours des années suivantes, surtout en ce qui concerne les biefs de marigot qui peuvent être repeuplés par la suite par des gîtes permanents passés inaperçus au cours des prospections préliminaires, ou par des gîtes mal assainis, ou par des poches d'eau traitées trop tardivement alors qu'un certain nombre de bulins se sont déjà enfoncés dans la vase du fond pour $y$ « estiver ».

Cependant, cette opération-pilote permet de donner un ordre de grandeur du coot d'une opération antimollusques dans une région où les foyers d'infestation à bilharziose sont des mares disséminées, parfois très éloignées les unes des autres, ou des bas-fonds de marigot de grande superficie.

Cette première expérience montre que le prix de revient du produit et le coût de la main-d'œuvre sont très faibles par rapport aux frais de déplacement occasionnés par le transport du molluscicide et du personnel chargé de l'opération.

En effet, il suffit de 20 à $60 \mathrm{~kg}$ de Zirame pour assainir une mare (prix du Zirame : 230 francs C. F. A. environ le $\mathrm{kg}$ ) et deux man'œuvres pour le répandre. Mais l'accès de ces mares esł si difficlle qu'une demi-journée est quelquefois nécessaire pour les aborder avec un véhicule tous terrains devant se frayer un passage à travers des zones boisées, où n'existe aucune piste, mais seulement des sentiers empruntés par les pasteurs.

\section{CONCLUSION}

En résumé, cette opération-pilote de prophylaxie antibilharzienne a montré :

$1^{\circ}$ qu'en eau calme, très chargée en matières organiques, le diméthyldithiocarbamate de zinc a une DL 100 sur B. guernes à des doses de 1 , d̀ 3 p. p. m.

$2^{\circ}$ que ce produit diffuse très bien, même dans des gîtes à mollusques encombrés par une flore abondante composée de Nymphaeo et de lotus.

$3^{\circ}$ que pour le traitement des mares de petite et moyenne dimensions, le Zirame peut être répandu à leur périphérie d'où économie de temps et de main-d'œuvre, mais que, par contre, 
dans les biefs de marigots longs et d'accès parfois difficile, le molluscicide doit être répandu à bord d'une pirogue ou mieux d'un canot pneumatique.

$4^{\circ}$ que le Zirame n'est que faiblement toxique pour les poissons existant dans ces points d'eau.

50 qu'il ne présente aucune toxicité pour le personnel chargé de son épandage, pas plus que pour les habitants ou les animaux utilisant les eaux traitées.

- Laboratorre national de f'élevage et de recherches vétérinaires de Dakar-Hann (Rép. du Sénégal) (Service d'helminthologis) - Ministère de la santé de la République du Sénégal (Service des grandes endémıes)

\section{SUMMARY}

Concerning a pilot trial on antibilharzia prophylaxy carried out with dimethyldithiocarbamale of zinc (Zirame)

A pilot triai on antibilharzia prophylaxy was conducted in eastern Senegal and proved:

1) that in still waters contanning high quantities of organic matter, dimethyldithiocarbamate of zinc has a L. D. 100 on B. guernei at dose from 1.5 to 3 p. p. m. ;

2) that this chemical spreads extremely well, even in molluscan habitats where the flora is composed of an abundance of nymphaea and lotus;

3) that for the treatment of small and medium sized ponds, Zirame may be spread on the periphery thus saving time and labour but, on the otherhand, for large sized ponds, the molluscicide must be spread from a canoe or, better still, from a rubber dinghy;

4) that Zirame is only feebly toxic to fish ;

5), that it has no toxic effects on the workers spreading it or on the animals and people using the treated water.

\section{RESUMEN}

\section{Acerca de una operacion piloto de profilaxia antibilharziana realizada por medio del dimetilditiocarbamato de zinc (Zirame)}

Una operación piloto de profilaxia antibilharziana, realizada en el Senegal oriental ha servido para demostrar :

1) que en agua calma, muy cargada de materia orgánica, el dimetilditiocarbamato de zinc a una D L 100 sobre $B$. guernei a dosis de 1,5 a 3 p. p. m. :

2) que este producto tiene una buena difusión, incluso en morada de moluscos atascadas por una flora abundante compuesta de Nynphoeo y de lotus ;

3) que para el tratamiento de las albercas de dimensiones pequeñas y medianas, el Zirame puede ser aplicado en su periferia, derivândose de ello una econamía de tiempo y de mano de obra pero que, contrariamente, el moluscicida debe ser aplicado a bordo de una piragua o, mejor aún, de una canoa neumática :

4) que el Zirame es poco tóxico para los peces que existen en estos puntos de agua ;

5) que nos presenta ninguna toxicidad para el personal encargado de su aplicación, del mismo modo que para los habitantes o animales que utilizan las aguas tratadas: 


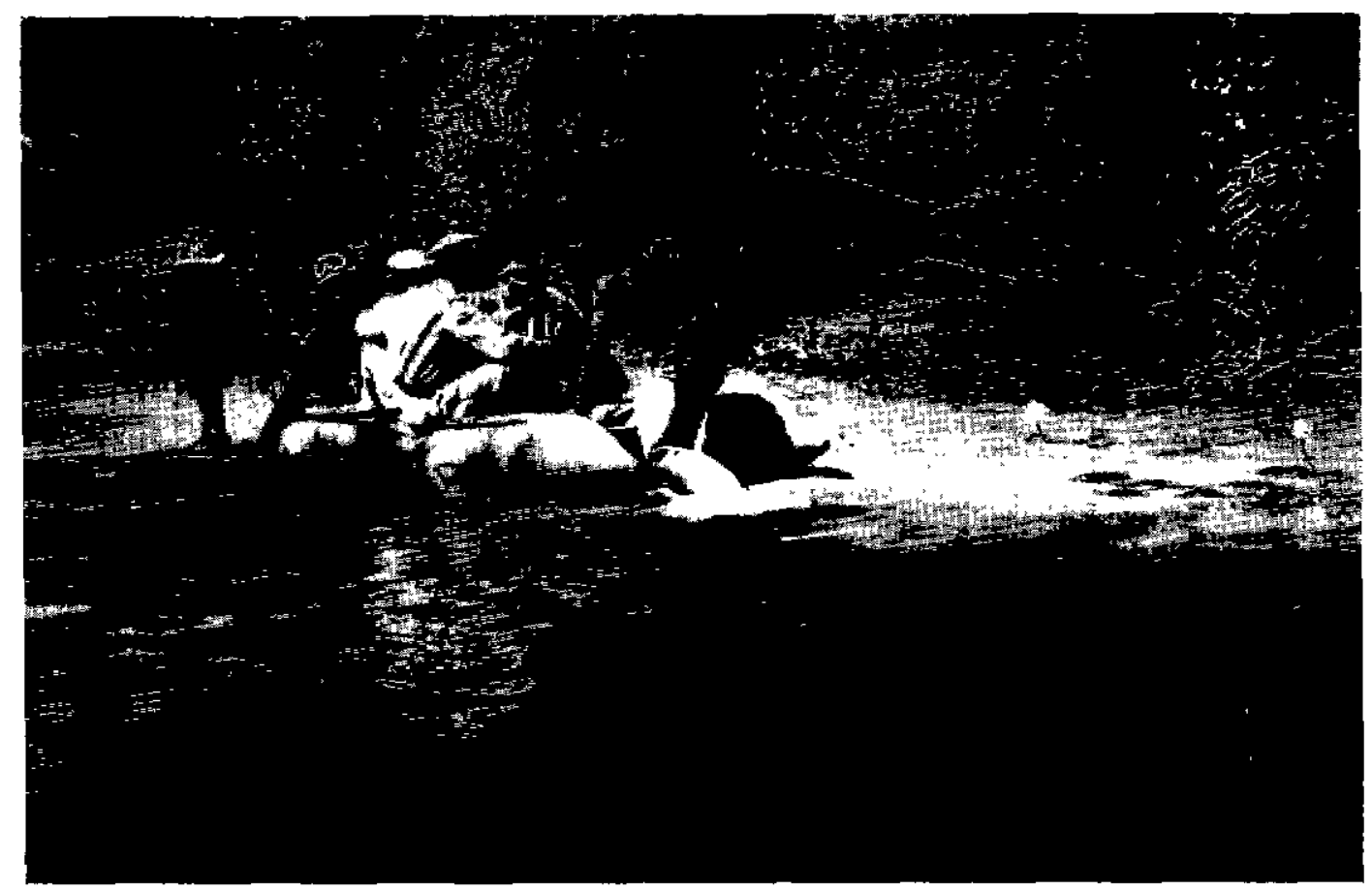

Photo 1. - Morıgot de Sinthiou Malémé. - L'équipe d'assainissement montée sur le canol pneumatıque franchit un passage de fable profondeur en pleine forêt. Les rames n'étant plus d'aucune utılité, un aide muni de boltes da chasse pousse le canot à la main.

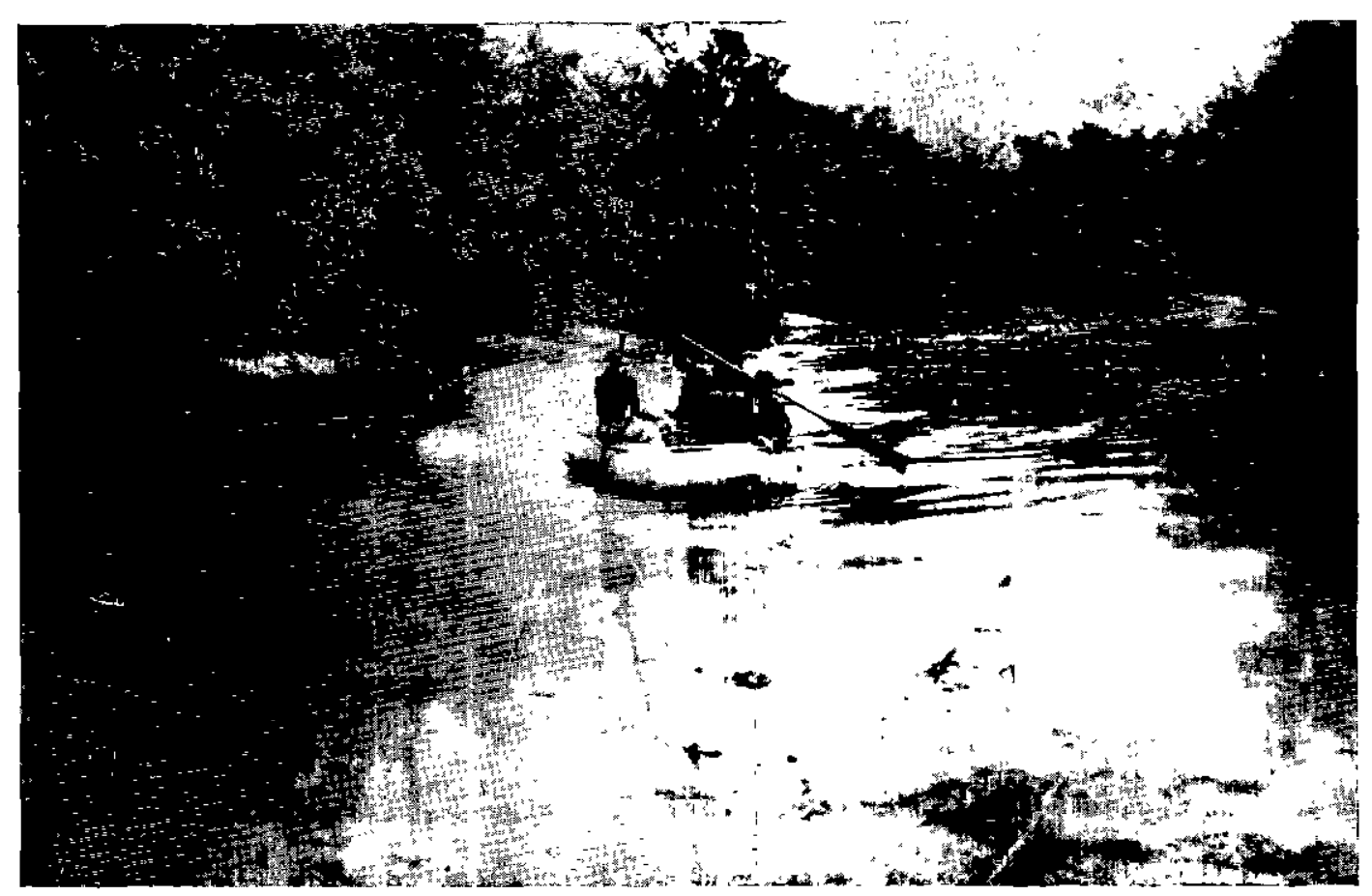

Photo 2. - Marigot de Maka - Sarakolé - Epandage de zirame. 


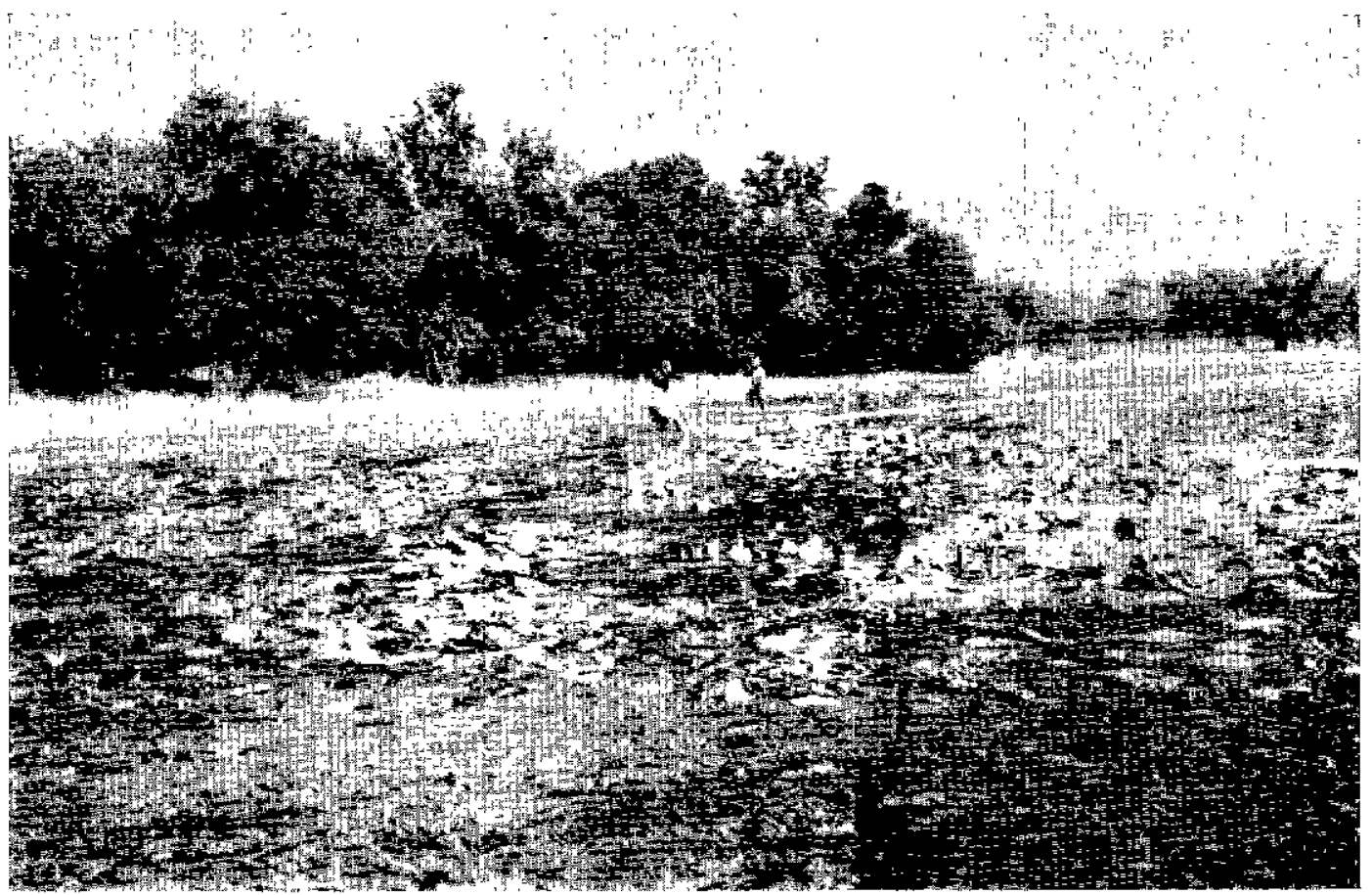

Photo 3. - Mare de Maka II. - Fond vaseux. Epandage de zırame à la périphérıe de la mare

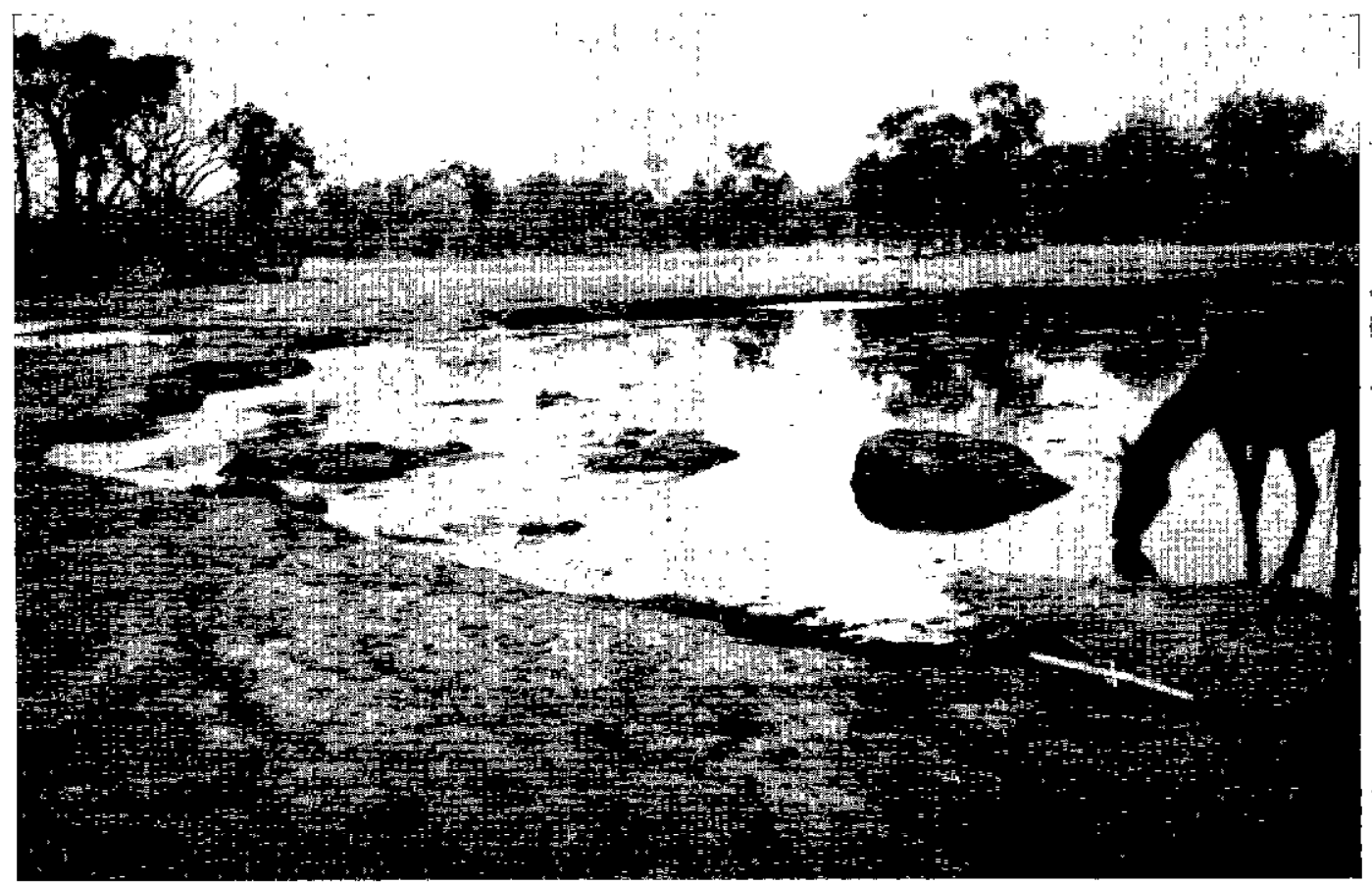

Photo 4. - Mare de Sill. Sol latéritique. Foyer important de bilharziose, 50 à 60 p. 100 des B. guernei infestés par des formes larvaires de Sch. curassoni. La présence de très nambreux crocodiles ne gêne en rien f'utilısation de ce point d'eau par les pasteurs transhumants et les habitants du village de Sill. 


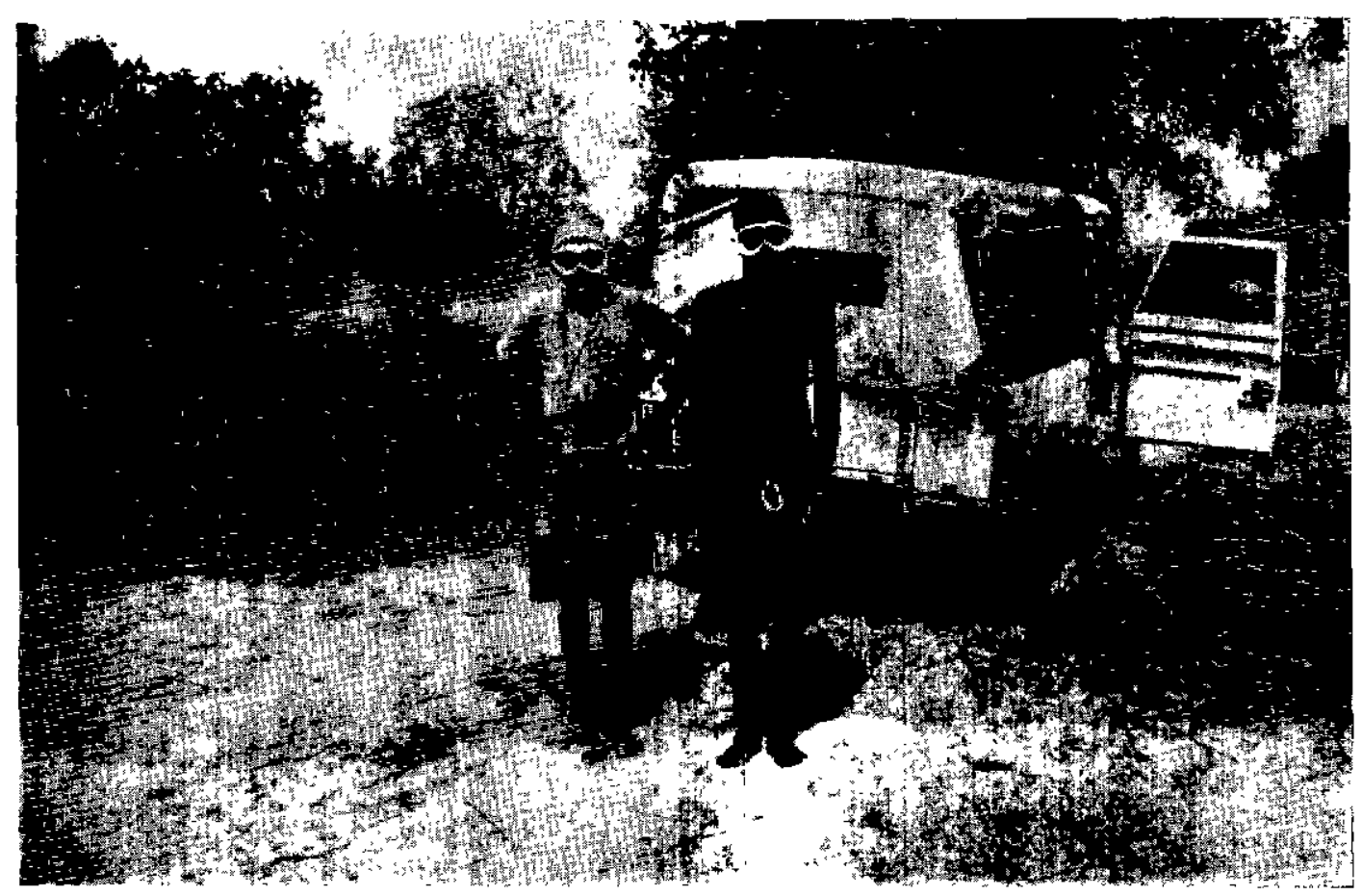

Photo 5. - L'équipe d'épandage de zirame en tenue de travail.

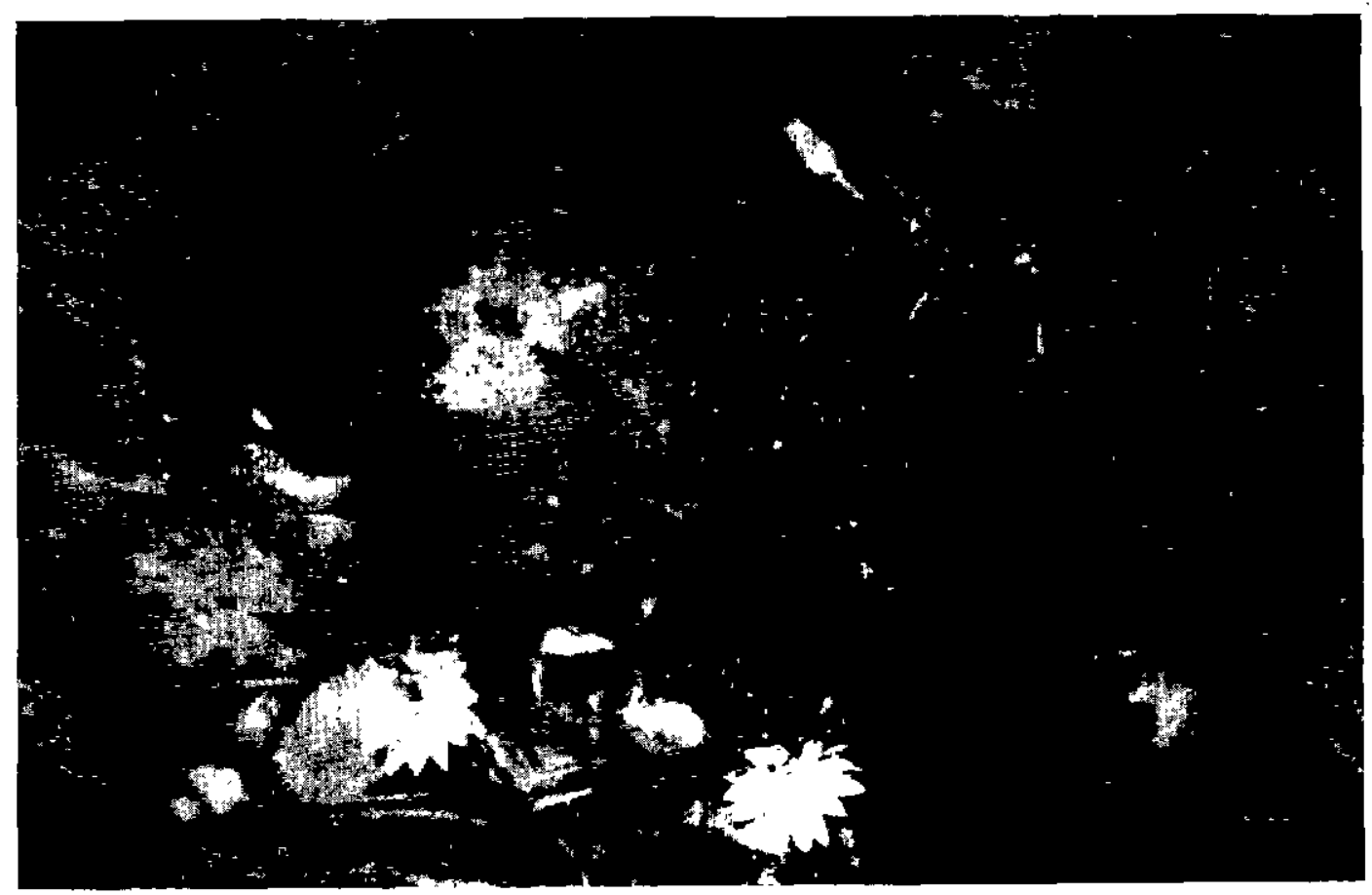

Photo 6. - More de Makil. - Pieds de nénuphars sur lesquels on aperçoit de très nombreusés coquilłes de Bulinus guernel lués par le zírame (contrôles faits 16 jours après l'épandage du pradult molluscicide). 


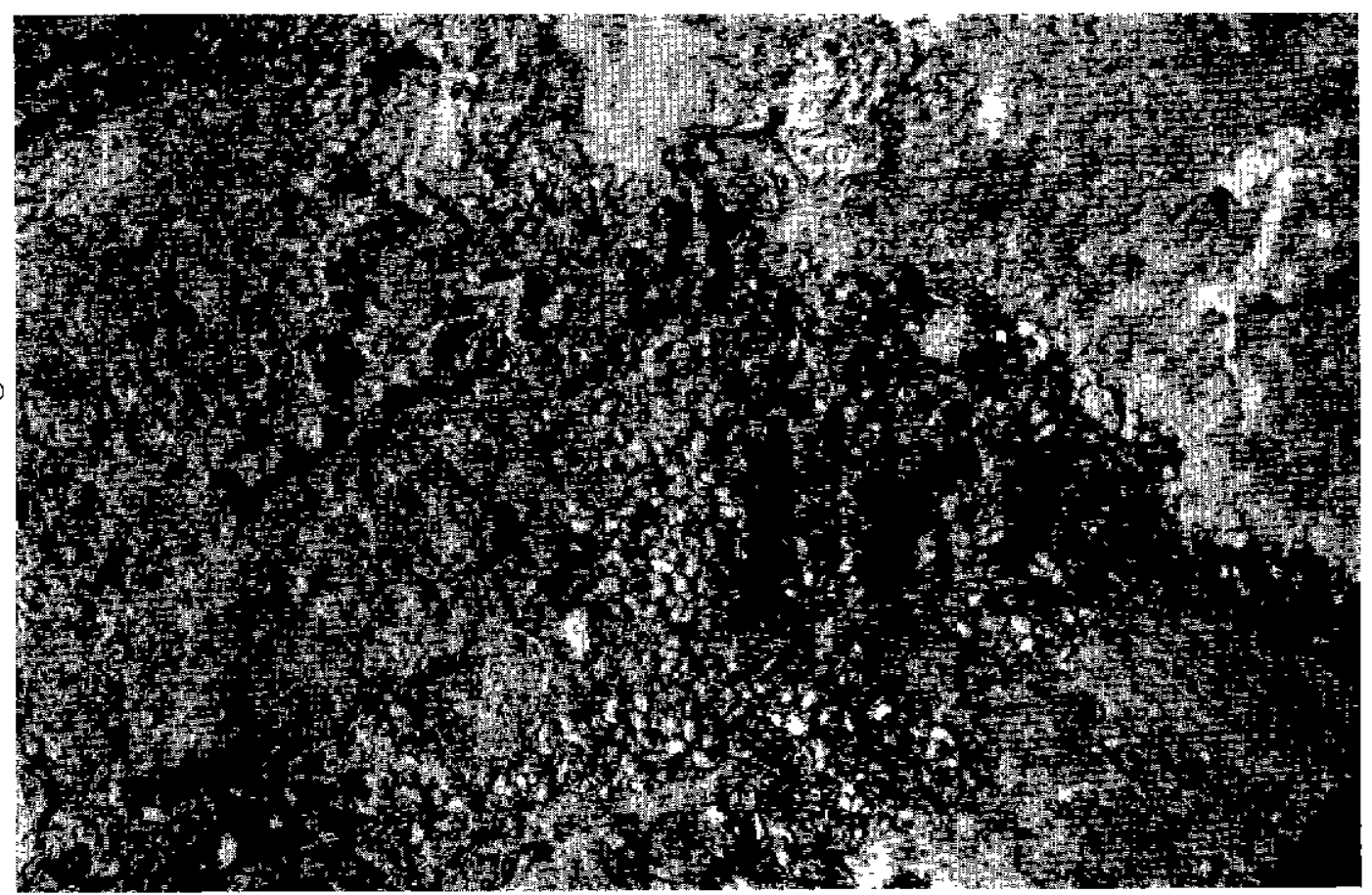

Photo 7. - Mare de Makil. - Coquilles vides de Bulinus guernei el Caelatura mesafrıcana tués par le zirame et reposant sur les bords latéritiques de la mare. Les contrôles d'efficacité du produit molluscıcide ayant été faits 16 jours après son épandage, les cadavres sont restés sur place alars que le niveau de l'eau balssaıt par assèchement progressif du paint d'eau.

\section{BIBLIOGRAPHIE}

1 GÖNNERT (R.). - Resultats of laboratory and field trials with the molluscicide Bayer 73. Bull. Org. mand. Santé. 1961, 25, 483-501.

2 GRETILLAT (S.). - Epidémiologie de la bilharziose vésicale au Sénégal-oriental. $\mathrm{Ob}$ servations sur l'écologie de Bulinus guernei et de Bulinus senegolensis. Bull. Org. mond. Sanié., 1961, 25, 459-66.

3 GRETILLAT (S.). - Un nouveau molluscicide, le diméthyldithiocarbamałe de zine (Zirame). Bull. Org. mond. Sonté, 1961, 25, $581-88$

4 GRETILLAT (S.). - Prophylaxie des affections d̀ trématodes de l'homme et des animaux domestiques par destruction des mollusques hôtes intermédiaires. Cahiers Méd. Vét., 1961, $30,153-71$.
5 GRETILLAT (S.). - Distomatose et bilharziose des ruminants domestiques. Leur prophylaxie par la lutte antimollusques. Rev. Elev. Méd. vét. Pays Tropicaux, 1961, 14, 3. 293-313.

6 GRETILLAT (S.). - Un molluscicide (Zirame) actif contre les formes larvaires de Culicidae. Bull. Org. mond. Santé 1962, 26, 67-74.

7 GRETILLAT (S.). - Recherches sur le cycle évolutif du schistosome des ruminants domestiques de l'Ouest africain (Schistosoma curassoni Brumpt, 1931): C. R. Acad. Sci., 1962, 255, 1657-59.

8 GRETILLAT (S.). - Une nouvelle zoonose, la «Bilharziose Ouest Africaine» à Schistosoma curassoni Brumpt, 1931, commune à l'homme ef aux ruminants domestiques. C. R. Acad. Sci., 1962, 255, 1805-7. 
9 GRETILLAT (S.). - Etude du cycle évolutif du schistosome des ruminants domestiques de l'Ouest africain et confirmation de l'espèce Schistosoma curassoni Brumpt, 1931. Ann. Parasif. Hum. Comp., 37, 4, 556-68.

10 NOLAN (M. O.) ef BOND (H. W.). - Results of laboratory screening tests of chemical compounds for molluscocidal activity - III - Derivatives of abietic acid., Amer. J. trop. Med. Hyg., 1955, 4, 152-55.
11 PAULINI (E.), CHAIA (G.) ef FreITAS (J. De R.). - Trials with the molluscicides Rhodiacid and Bayer 73. Bull. Org. mond. Santé, 1961, 15, 706-09.

12 SCHIFF (C. J.). - Trials with a new molluscicide, Bayer 73, in Southern Rhodesia. Bull. Org. mond. Sonté, 1961, 15, 533-42.

13 WEBBE (G.). - Laboratory and field trials of a new molluscicide Bayer. 73, in Tanganyika. Bull. Org. mond. Santé, 1961, 15, 525-31. 